\title{
Correction to: Imaging the Ethiopian Rift Region Using Transdimensional Hierarchical Seismic Noise Tomography
}

\author{
Addis Eshetu, ${ }^{1,2}$ (D) Tilahun Mammo, ${ }^{1}$ and Frederik Tilmann ${ }^{3,4}$
}

Correction to: Pure Appl. Geophys. 178 (2021), 4367-4388

https://doi.org/10.1007/s00024-021-02880-2

The URL for the supplementary dataset has been revised. Instead of

https://dataservices.gfz-potsdam.de/panmeta works/review/c5b7a3d9a5f2c476067b1ae4f91cf2 96929d55e980e8b522fbf92e48844bf068/, the correct URL is as follows:
https://doi.org/10.5880/GFZ.2.4.2021.007.

The original article has been corrected.

Publisher's Note Springer Nature remains neutral with regard to jurisdictional claims in published maps and institutional affiliations.

The original article can be found online at https://doi.org/10.1007/ s00024-021-02880-2.

1 School of Earth Sciences, Addis Ababa University, Addis Ababa, Ethiopia. E-mail: addisewku@gmail.com

2 Department of Mathematics, Wolkite University, Wolkite, Ethiopia.

3 Deutsches GeoForschungsZentrum GFZ, Telegrafenberg, 14473 Potsdam, Germany.

4 Department of Geosciences, Freie Universität Berlin, Berlin, Germany. 